\title{
Back Propagation Wavelet Neural Network Based Prediction of Drill Wear from Thrust Force and Cutting Torque Signals
}

\author{
$\mathrm{Xu}$ Yang (Corresponding author) \\ Department of Mechanical System Engineering, Faculty of Engineering, Gunma University \\ 1-5-1 Tenjin-cho, Kiryu 376-8515, Japan \\ Department of Mechanical Engineering, Dalian Polytechnic University, Dalian 116034, China \\ E-mail: yangxv1994@163.com \\ Hiroyuki. Kumehara \\ Department of Mechanical System Engineering, Faculty of Engineering, Gunma University, 1-5-1 \\ E-mail: kumehara@me.gunma-u.ac.jp \\ Wei Zhang \\ Department of Mechanical Engineering, Dalian Polytechnic University, Dalian 116034, China \\ E-mail: dlzhangwei@yahoo.com
}

\begin{abstract}
The fast monitoring of tool wears by using various Cutting signals and the prediction models developed rapidly in recent years. Comparatively, various wear forecast models based on artificial neural networks (ANN) perform much better in accuracy and speediness than the conventional prediction models. Combining the prominent dynamic properties of back propagation neural network (BPNN) with the enhanced ability of a wavelet neural network (WNN) in mapping nonlinear functions, a Back propagation wavelet neural network (BPWNN) is newly established to perform prominent prediction of drill wear. In this work, a multilayer BPWNN with wavelet algorithm has been applied to predict the average wear of a K10 carbide drill bit for drilling on a high silicon aluminum work piece. Mean value of the thrust force, cutting torque, and drilling depth, spindle speed and feed-rate are inputs to the network, and drill wear is the output. Drilling experiments have been carried out over a wide range of cutting conditions and the effects of drill wear, cutting conditions (spindle speed, drilling depth and feed-rate) on the thrust force and cutting torque have been investigated. Performance of BPWNN has proved to be satisfactory by experimental result. The accuracy of the prediction of drill wear using BPWNN is found to be better than using BPNN, and that BPWNN can learn the pattern faster compared to BPNN and could be used advantageously in online drill wear monitoring and prediction.
\end{abstract}

Keywords: Back propagation wavelet neural network, Drilling, Thrust force, Cutting torque, Drill wear monitoring

\section{Introduction}

Manufacturing industries are trying to reduce the operation cost and better quality of product. In machining process, tool wear and breakage often has a fatal effect on the product. So, automation with online monitoring and prediction in metal cutting operations is a new approach toward improvement of the quality of the product as well as reduction of the cost of the product. Especially prediction of tools wear plays a very significant role to realize a automated manufacturing system. Drilling represents over 30\% of all cutting operations performed in industries (Young Jun Choi, Min Soo Park, Chong Nam Chu, 2008). Monitoring and prediction of drill wear is an important issue since wear on drill affect the hole quality and tool life of the drill. Therefore, monitoring and prediction of drill wear is an important area of research. For improving the performance of decision-making in tool condition monitoring, many works have been reported in the broad field of different type of intelligent monitoring systems.

Tool wear detection methods can be direct or indirect. Indirect methods, in which various signals of sensors (thrust force and torque, current, acoustic emission, vibration) correlating to tool wear have been extensively applied to prediction of tool wear detection (E. Jantunen, 2002). Mauri and Matti (R. Mauri, S Matti, 1995) reported that the online measurements of cutting power, spindle motor current, torque and thrust force did not vary significantly in entire 
tool life until the failure of the drill bit. $\mathrm{Li}$ and Wu (T.I. Liu, S.M. Wu, 1990) introduced thrust force and torque which were selected as the features relevant to the four drills wear states. Bhattacharyya and Sengupta (L. Xiaoli, S.K. Tso, 1999) established the combinations of signal processing techniques for real-time estimation of tool wear in face milling using cutting force signals are presented. Xiaoli and Tso (P. Bhattacharyya, D. Sengupta, S. Mukhopadhyay, 2007) established the correlation between drill wear and motor current at different cutting conditions. Xiaoli (L. Xiaoli, 1999) applied wavelet transform on motor current signals to detect the breakage of a small diameter drill. AE has been used to tool monitoring successfully during turning operations (E. Govekar, J. Gradisek, I. Grabe, 2000). Abu-Mahfouz (I. Abbu-Mahfouz, 2003) reported that the drilling wear detection and classification uses vibration signals and artificial neural network. Parameters for monitoring drill conditions, thrust force and cutting torque have been used because they are the most sensitive to the changes of drill conditions (G.S. Li, W.S. Lau, Y.Z. Zhang, 1992).

Artificial neural network can effectively map the non-linear relationship of the sensors signals and wear state. Multilayer neural network with back propagation learning, a simple, robust and non-linear modeling device, has been widely applied to develop systems for monitoring wear of turning, milling and drilling tools (D.E. Dimla, P.M. Lister, N.J. Leighton, 1997), Hong et al. (G.S. Hong, M. Rahaman, Q. Zhou, 1992) used cutting force signal input to back propagation neural network for tool wear monitoring. Lin and Ting (S.C. Lin, C.J. Ting, 1996) used back propagation neural network, and determined the best architecture for drill wear predicting. Panda and Singh (S.S. Panda, A.K. Singh, D. Chakraborty, S.K. Pal, 2006) introduced a method to chip thickness as an additional input parameters entered into the neural network to obtain a better training effect. Panda and Chakraborty (S.S. Panda, D. Chakraborty, S.K. Pal, 2008) used thrust force, torque and vibration the input to two different types of different back propagation neural network (BPNN) and radial basis function network (RBFN) for predicting flank wear in drills.

Wavelet neural network (WNN) is a feed forward neural network based on wavelet transform (WT) (H. Zhong, J. Zhang, M. Gao, J. Zheng, G. Li, L. Chen, 2001). This successful synthesis of theories has generated a new class of networks called Wavelet Neural Networks (WNN) (Q. Zhang, A. Benveniste, 1992). The distinct feature of these networks is that they use wavelet functions as the activation functions of hidden layer neurons. Using theoretical features of wavelet transform, network construction methods could be developed (Szu H, Telfer B, Kadambe S., 1992). The network has advantages of the wavelet transform (in denoising, background reduction and recovery of characteristic information) and also has neural network capacity of "universal" approximation (H. Zhong, J. Zhang, M. Gao, J. Zheng, G. Li, L. Chen, 2001).

The present paper, effects of cutting conditions (spindle speed, drilling depth and feed-rate) and drill wear on thrust force and cutting torque has been tested; and the drilling process was modeled by a neural network with a back wavelet algorithm and weights gradient descent method. Comparison has also been made between BPNN and BWFN in drill wear monitoring. The performance of BPWNN has been found to be satisfactory while validated with experimental result.

\section{Back propagation wavelet neural network}

\subsection{Back propagation wavelet neural network architecture}

Back propagation wavelet neural network (BPWNN) is an ANN that is integrated with wavelet techniques and has been used successfully in many fields. Instead of conventional nonlinear sigmoid transfer functions, the transfer function of the nodes in a wavelet neural network is wavelet bases. Because wavelet bases have the characteristics of time precision in high frequency domains and frequency precision in low frequency domains due to dilating and translating the mother wavelet, the ability of a WNN in mapping complicated nonlinear functions is enhanced considerably.

BPWNN has been used in the present work. Basic structure of BPWNN with a single hidden layer is shown in Figure 1. Functioning in three stages, namely learning or training or testing. The network consists of three layers: an input layer, an hidden layer, and an output layer. Input layer receives information from the external sources to the network for processing. Hidden layer receives information from the input layer, and processes the information, and output layer receives processed information from the network, and sends the results out to an external receptor. Where, $W_{j i}$ is the weight between $i$ th neuron of the input layer and $j$ th neuron of the hidden layer, $V_{k j}$ the weight between $j$ th neuron of the hidden layer and $k$ th neuron of the output layer, $\theta_{j}$ the bias at $j$ th neuron of the hidden layer, $\tau_{k}$ the bias at $k$ th neuron of the output layer, respectively.

Back propagation wavelet neural network algorithm has been used in the present work. And thrust force, torque, drilling depth, spindle speed and feed-rate are used as input parameters and corresponding wear are used as the output parameter as showing in Figure 1. The normalized data sets are used for training the network. The data sets are normalized in the range of $0.1-0.9$ by using Eq. (1): 
$I_{i}=\frac{0.8}{d_{\max }-d_{\min }}\left(d_{i}-d_{\min }\right)+0.1$

Where $d_{i}$ is the actual value, $d_{\max }$ is the maximum value of $d_{i}, d_{\min } \min$ is the minimum value of $d_{i}$, and $I_{i}$ is the normalized value corresponding to $d_{i}$.

Outputs signal of input layer are modified by interconnection weight $\left(W_{j i}\right)$ of $i$ th node of input layer to $j$ th node of Hidden layer. The sum of the inputs signals is then modified by a Morlet transfer function. $(\psi)$. Similarly, outputs signal of hidden layer are modified by interconnection weight $\left(V_{k j}\right)$ of $j$ th node of hidden layer to $k$ th node of output layer. The sum of the modified signal is then modified by sigmoid transfer function $(f)$ and output is collected at output layer.

Let $I_{l}^{P}=\left(I_{1}^{P}, I_{2}^{P}, \cdots \cdots, I_{i}^{P}, \cdots \cdots, I_{l}^{P}\right)$ is the $i$ th input data from the $P$ th training pattern of the data set to the input layer. $l$ is the total number of input parameter applied to the network.

Output from a neuron in the input layer by using Eq. (2):

$O_{i}^{P}=I_{i}^{P} \quad i=1,2, \cdots \cdots l$

Output from a neuron in the hidden layer by using Eq. (3):

$O_{j}^{P}=\psi\left(\sum_{i=0}^{l} \frac{W_{j i} O_{i}^{P}-b_{j}}{a_{j}}+\theta_{j}\right), j=1,2, \cdots \cdots, m$

Output from a neuron in the output layer by using Eq. (4):

$O_{k}^{P}=f\left(\sum_{i=0}^{l} V_{k j} O_{j}^{P}+\tau_{k}\right), k=1,2, \cdots \cdots, n$

Suppose $\psi(x) \in L^{2}(R)$ is a mother wavelet, a series of daughter wavelets can be developed through dilating and translating function $\psi(x)$ :

$\psi_{a, b}(x)=\frac{1}{\sqrt{|a|}} \psi\left(\frac{x-b}{a}\right)$

Where $a$ is the dilation factor, $b$ is the translation factor.

Mother wavelet is suitable for this work to adopt the wavelet as the transfer function of the BPWNN in consideration of high resolution in both time and frequency domains. The Morlet wavelet is defined as follows (Szu H, Telfer B, Kadambe S, 1992):

$\psi(x)=\cos (\omega \cdot x) \times \exp \left(-x^{2} / c\right)$

In Hidden layer transfer function $(\psi)$ the signal function does not undergo wavelet transformation and inverse transformation. Therefore, it is applicable in this work to use the type of Morlet wavelet as follows (Eq. (6)):

$\psi(x)=\cos (1.75 x) \times \exp \left(-x^{2} / 2\right)$

Output layer transfer function $(f)$ of sigmoid transfer function as follows (Eq. (7)):

$f(x)=\frac{1}{1+\exp (-x)}$

\subsection{Learning or training in Back propagation wavelet neural network}

Batch mode type of supervised learning has been used in the present case, where, interconnection weights are adjusted using delta rule algorithm after sending the entire training sample to the network (H. Zhong, J. Zhang, M. Gao, J. Zheng, G. Li, L. Chen, 2001). During training, the predicted output is compared with the desired output, and the mean square error is calculated. If the mean square error is more than a prescribed limiting value, it is back propagated from output to input, and weights are further modified till the error or number of iterations is within a prescribed limit.

Mean square error (MSE), $E_{P}$ for pattern $P$ is defined as

$E_{P}=\frac{1}{2} \sum_{k=1}^{n}\left(Y_{k}^{P}-O_{k}^{P}\right)^{2}$ 
Where $Y_{k}^{P}$ is the target output, $O_{k}^{P}$ is the computed output.

At any epoch $\eta$, the gradient descent algorithm is well known, the modified with the adaptive learning factor and the momentum factor, can be summarized as the adjustment of the weight change according to Eq. (9) and Eq. (10).

$\Delta W(n+1)=\alpha \times \Delta W(n)-\eta \times \frac{\partial E_{P}}{\partial W}$

$\Delta V(n+1)=\alpha \times \Delta V(n)-\eta \times \frac{\partial E_{P}}{\partial V}$

$\eta$ is the learning rate, $0<\eta<1 . \alpha$ is the momentum coefficient, $0<\alpha<1$.

In the present case, prediction has been considered for training of the wavelet neural network, which consists of the following steps:

1) Training samples are normalized and read.

2) Weights of hidden layer and output layer are initialized to randomly within [0,1] values, and bias of hidden layer and output layer are initialized to randomly within $[0,1]$ values, and the dilation and translation factors $a$ and $b$ as well.

3) Determine other initial parameters as the following through trial and error. The momentum factor is fixed on 0.5-0.9. The initial learning rate is set at $0.3-0.9$, respectively. The stop conditions are the limitation of the mean square error (MSE) of 0.00003 and the maximum training iteration epochs of 2000.

4) Output from hidden layer was calculated as per Eq. (3). Output from output layer was calculated as per Eq. (4).

5) MSE of training sample was calculated. If the MSE training not reach the goal specified then weight is updated based on gradient descent method. The weight was updated in the present case in batch mode.

6) The process was carried out for a designated number of iteration.

\subsection{Testing of Back propagation wavelet neural network}

An experimental data set is divided into the training set and the testing set. The error on the testing set is monitored during the training process. The testing error is decreasing during the training. When the testing error increases to specified number of iterations, the training will stop. The weights when getting the minimum value of the testing error are returned.

\section{Experimental set-up}

The schematic diagram of the experimental setup is shown in Figure 2 The experiments were performed on a 5 -axis CNC vertical drilling machining center (MAKINO MC1010-5XA). The thrust force and cutting torque are recorded through a piezoelectric drilling dynamometer (YDZ-II02). Measuring range of thrust force varies from -5 to $5 \mathrm{kN}$ with a sensitivity of $3.8 \mathrm{pC} / \mathrm{N}$. Similarly range of torque varies from-100 to $100 \mathrm{Nm}$ with a sensitivity of $1.68 \mathrm{pC} / \mathrm{Ncm}$. Signals from the dynamometer were passed through low pass filter, amplified through charge amplifier (YE5850), and stored in the computer through a data acquisition system (NR-2000). The analog outputs from the sensors were converted to digital signals by A/D board of data acquisition system sends to personal computer (PC).

In the experiments, three types of drill flank( i.e. Multifacet I flank, Multifacet II flank and Standard flank) are considered. The Multifacet I, Multifacet II and Standard drills are shown in Figure 3. Scanning Electron Microscope has been used to measure the drill wear. Figure 4 shows the photographs of flank faces and corresponding wear of three drill's flanks (a) Multifacet I , (b) Multifacet II (c) Standard in the drilling experiments. Carbide drill bits with different flank have been used for drilling in high silicon aluminum alloy workpiece under different cutting conditions. A series of tests were carried out under different cutting conditions to confirm the effectiveness of the proposed method. The experiment conditions are shown in Table 1.

\section{Experimental results}

Drilling operations have been conducted over a wide range of cutting condition. Spindle speed has been varied in the range of 3000-6000 rpm in six steps. Feed rate has been varied from 0.1 to $0.2 \mathrm{~mm} / \mathrm{rev}$ in three steps. Drilling depth has been varied from 10 to $20 \mathrm{~mm}$ in three steps. K10carbide drills of three different Flank figures (standard, Multifacet I and Multifacet II) have been used for drilling in high silicon aluminum alloy A390 plates. Different combinations of three design variables viz. spindle speed; feed rate and drilling depth have been used to perform 18 different drilling operations on A390 plate. For each of these conditions, thrust force and torque have been measured using dynamometer and the data are stored in the computer through the WAVE SHOT! 2000 software. Corresponding to each cutting condition, the wear has also been measured using scanning electron microscope. The results of the 
experiments are tabulated in Table 2, which shows the thrust force, torque and the drill wear corresponding to 18 different cutting conditions.

\subsection{Effect of Input parameters on thrust force}

Figure 5-Figure 7 shows the effect of important cutting parameters on thrust force and torque during drilling operation.

Figure 5 shows variation of thrust force and torque with spindle speeds (3000 and 4000rpm) for different feed rate and different flank drill. It could also be observed that the decreases trend of thrust force and torque with Spindle speed is more pronounced at flank of Standard (feed rate $0.1 \mathrm{~mm} / \mathrm{rev}$ ) and Multifacet II (feed rate $0.2 \mathrm{~mm} / \mathrm{rev}$ ). Similarly it could be observed that thrust force and torque decreases with increase in spindle speed and this is due to facts that increase in spindle speed increase the temperature generation during shearing action of cutting tool, and hence it softens the material of the work piece which results in the reduction of the thrust force. While on the contrary be observed that the increasing trend of thrust force and torque with Spindle speed is more pronounced at flank of Multifacet I (feed rate $0.15 \mathrm{~mm} / \mathrm{rev}$ ), Because the extend of the cutting edge, the cutting temperature of Multifacet I drills is not increases with the increment of the rotating speed obviously.

Figure 6 shows variation of thrust force and torque with spindle speeds (4500 and 5500rpm) for different feed rate and different flank drill. It could also be observed that the decreases trend of thrust force and torque with Spindle speed is more pronounced at flank of Standard (feed rate $0.2 \mathrm{~mm} / \mathrm{rev}$ ) and Multifacet II (feed rate $0.15 \mathrm{~mm} / \mathrm{rev}$ ). While on the contrary be observed that the increasing trend of thrust force and torque with Spindle speed is more pronounced at flank of Multifacet I (feed rate $0.1 \mathrm{~mm} / \mathrm{rev}$ ), the condition in the range of 4500 and $5500 \mathrm{rpm}$ spindle speeds is same with 3000 and 4000rpm spindle speeds. Because the extending of the cutting edge, the cutting temperature of Multifacet I drills does not increase with the increment of the rotating speed obviously.

Figure 7 shows variation of thrust force and torque with spindle speeds (5000 and 6000rpm) for different feed rate and different flank drill. It could also be observed that the increasing trend of thrust force and torque with Spindle speed is more pronounced at flank of all. Because increase of cutting temperatur was not obvious, it doesn't soften the material,so it leads the increment of the thrust force and torque.

The thrust force, torque and the drill wear which correspond to cutting conditions are shown in Table 2 , it could be observed that the cutting parameters with the drill wear have been at nonlinear mapping relations. Combining the prominent dynamic properties of back propagation neural network with the enhanced ability of a wavelet neural network in mapping nonlinear functions, a back propagation wavelet neural network is newly established in this work to perform prominent prediction of drill wear.

\subsection{Drill wears monitoring and prediction by neural network}

In this case, the network has five input nodes and one output node. Neural network architectures, prepared using various combinations of input parameters (Table 2), such as spindle speed, feed rate, drilling depth, thrust force, torque, and with flank wear as the only output of the network. From the 18 data sets obtained in the experiment, 10 have been selected at front for training the network and remaining 8 are used for testing. Optimum network architecture include number of hidden layers, number of neurons in the hidden layers, learning rate $(\eta)$ and momentum coefficient ( $\alpha$ ) has been obtained by error based on mean square error MSE in training, MSE in testing, and convergence rate (number of iterations).

\subsubsection{Optimum neural network architecture of BPWNN and prediction performance}

Several architectures of the BPWNN are trained for different learning rates and momentum coefficient values. Performances of some of the architectures for ten training and eight testing result are shown in Table 3 . Best network architecture has been arrived at by trial and error of different combination of $(\eta)$ and momentum coefficient ( $\alpha$ ) of hidden nodes. In the present case, based on these observations, based on these observations, the optimum network obtained is 5-15-1 with $\alpha=0.9$ and $\eta=0.8$.

Figure 8 shows the variation of MSE in training and testing with number of iterations for network of 5-15-1 with $\alpha=0.9$ and $\eta=0.8$. It could be observed that network could be trained till 38 iterations after which it starts over fitting and at this point MSE for training as 0.0000274 and MSE for as testing 0.0000051.

\subsubsection{Optimum neural network architecture of BPNN and prediction performance}

The BPNN apply architectures are similar to those of the BPWNN described in Table 3, it is BP networks of 5-input model for drill wear forecasts, the activation functions are all sigmoid without wavelet.

Monitoring amd prediction performances of BPNN of the architectures for ten training and eight testing result are shown in Table 4. In the present case, the optimum network obtained is 5-5-1 with $\alpha=0.9$ and $\eta=0.8$.

Figure 9 shows the variation of MSE in training and testing with number of iterations for network of 5-5-1 with $\alpha=0.9$ and $\eta=0.8$. It could be observed that network could be trained till 52 iterations after which it starts over 
fitting and at this point MSE for training as 0.0000483 and MSE for testing as 0.0000064 .

\subsection{Comparison of monitoring amd prediction performances between BPNN and BPWNN}

The BPWNN has been trained; it has been verified with testing sample. Figure 10 is optimum to choose the architecture of BPWNN. It shows percentage of error between actual value and the predicted value, it can be observed that the present 5-15-1 BPNN $\alpha=0.9$ and $\eta=0.8$ predicts the result within $9.38 \%$. Similarly, Figure 11 is optimum to choose the architecture of BPNN. It expresses percentage of error between actual value and the predicted value and it can be observed that the present 5-5-1 BPNN $\alpha=0.9$ and $\eta=0.8$ predicts the result within $12.31 \%$.

Figure 12 shows network testing set by performing a linear regression between each value of the network response and the corresponding target. It gives similar comparisons clearly.

\section{Conclusions}

When combination of the process parameters such as drilling depth, spindle speed, feed is supplemented with sensors signals such as thrust force and torque for training a Back propagation wavelet neural network, The enhanced basis function network is used to predict drill wear. It has been showed in the work that both BPNN and WNN can predict the drill wear reasonably well. From the present work, the following specific conclusions have been drawn:

Inclusion of thrust force, torque signals, drilling depth and Cutting conditions as input to train neural network results in a well-trained network, which can predict the wear with prominent accuracy.

This paper presents a new model for prediction drill wear based on the Back propagation wavelet neural network (BPWNN) and weights are adjusted using gradient descent method. Experiments prove that the model is capable of mapping drill wear that is usually highly nonlinear. This is because the BPWNN combines the advantages of both the BPNN and WNN.

Experiments show that the value of the prediction MSE by the trained BPWNN described in the paper is less than those by other typical models (BPNN). It proves that the effort for improving the prediction precision in the paper is fruitful, and BPWNN can monitor online drill wear faster than BPNN.

\section{References}

D.E. Dimla, P.M. Lister, N.J. Leighton, (1997). Neural network solutions to the tool condition monitoring problem in metal cutting - a critical review of methods, International Journal of Machine Tools \& Manufacture. 37 (9) (1997). 1219-1241.

E. Govekar, J. Gradisek, I. Grabe, (2000). Analysis of acoustic emission signals and monitoring of machining processes, Ultrasonic .38 (2000). 598-603.

E. Jantunen, (2002). A summary of methods applied to tool condition monitoring in drilling, International Journal of Machine Tools \& Manufacture. 42 (2002) 997-1010.

G.S. Hong, M. Rahaman, Q. Zhou, (1996). Using neural network for tool condition monitoring based on wavelet decomposition, International Journal of Machine Tools \& Manufacture. 36 (5) (1996) 551-566.

H. Zhong, J. Zhang, M. Gao, J. Zheng, G. Li, L. Chen, (2001). Chemom. Intell. Lab. Syst.59 (2001) 67.

I. Abbu-Mahfouz, (2003). Drilling wear detection and classification using vibration signals and artificial neural network, International Journal of Machine Tools \& Manufacture. 43(2003) 707-720.

L. Xiaoli, (1999). On-line detection of the breakage of small diameter drills using current signature wavelet transform, International Journal of Machine Tools \& Manufacture. 39 (1999) 157-164.

L. Xiaoli, S.K. Tso, (1999). Drill wear monitoring based on current signals, Wear 231 (1999) 172-178.

P. Bhattacharyya, D. Sengupta, S. Mukhopadhyay, (2007). Cutting force-based real-time estimation of tool wear in face milling using a combination of signal processing techniques, Mechanical Systems and Signal Processing 21 (2007) 2665-2683.

Q. Zhang, A. Benveniste, (1992). IEEE Trans. Neural Network.3 (1992) 889.

R. Mauri, S Matti, (1995). Tool wear and failure in the drilling of stainless steel, Journal of Materials Processing Technology. 52 (1995) 35-43.

S. Li, W.S. Lau, Y.Z. Zhang, (1992). In-process drill wear and breakage monitoring for a machining center based on cutting force parameters, International Journal of Machine Tools and Manufacture. 32 (1992) 855-867.

S.C. Lin, C.J. Ting, (1996). Drill wear monitoring using neural network, International Journal of Machine Tools \& Manufacture. 36 (4) (1996) 465-475.

S.S. Panda, A.K. Singh, D. Chakraborty, S.K. Pal, (2006). Drill wear monitoring using back propagation neural network, 
Journal of Materials Processing Technology 172 (2006) 283-290.

S.S. Panda, D. Chakraborty, S.K. Pal, (2008). Flank wear prediction in drilling using back propagation neural network and radial basis function network, Applied Soft Computing 8 (2008) 858-871.

Szu H, Telfer B, Kadambe S, (1992). Neural network adaptive wavelets for signal representation and classification. Opt Eng 1992; 36(9): 1907-1916.

T.I. Liu, S.M. Wu, (1990). On-line detection of drill wear, ASME Journal of Engineering for Industry. 112 (1990) 299-302.

Young Jun Choi, Min Soo Park, Chong Nam Chu, (2008). Prediction of drill failure using features extraction in time and frequency domains of feed motor current, International Journal of Machine Tools \& Manufacture.48 (2008) 29-39.

Table 1. Cutting conditions

\begin{tabular}{ll}
\hline Tool & Drill type: Standard flank, Multifork I flank and Multifork II flank \\
& Diameter: $8 \mathrm{~mm}$ \\
& Tool material: K10carbide \\
& TiAlN coated \\
\hline Cutting conditions & Spindle speed: $3000,4000,4500,5000,5500$ and $6000 \mathrm{rpm}$ \\
& Feed-rate: $0.1,0.15 \mathrm{and} 0.2 \mathrm{~mm} / \mathrm{rev}$ \\
& Exterior and interior lubrication \\
Work-piece & High silicon aluminum alloy A390 \\
\hline
\end{tabular}

Table 2. Experimental input/output data for neural network

\begin{tabular}{llllllll}
\hline Serial number & Drill type & Speed $(\mathrm{rpm})$ & Feed $(\mathrm{mm} / \mathrm{rev})$ & Deepness $(\mathrm{mm})$ & Thrust force $(\mathrm{N})$ & Torque $(\mathrm{Nm})$ & Wear(mm) \\
\hline 1 & Standard & 3000 & 0.1 & 100 & 2148.04 & 10.34 & 0.178 \\
2 & Multifork I & 3000 & 0.15 & 150 & 4155.97 & 15.68 & 0.093 \\
3 & Multifork II & 3000 & 0.2 & 200 & 3032.05 & 15.35 & 0.176 \\
4 & Standard & 4000 & 0.1 & 150 & 1983.18 & 9.32 & 0.157 \\
5 & Multifork I & 4000 & 0.15 & 200 & 4607.97 & 17.6 & 0.084 \\
6 & Multifork II & 4000 & 0.2 & 100 & 2876.34 & 14.63 & 0.061 \\
7 & Multifork I & 4500 & 0.1 & 100 & 2972.67 & 11.81 & 0.097 \\
8 & Multifork II & 4500 & 0.15 & 150 & 2090.95 & 10.04 & 0.113 \\
9 & Standard & 4500 & 0.2 & 200 & 3125.68 & 11.97 & 0.25 \\
10 & Multifork II & 5000 & 0.1 & 200 & 1740.67 & 7.19 & 0.137 \\
11 & Standard & 5000 & 0.15 & 100 & 2318.48 & 8.84 & 0.136 \\
12 & Multifork I & 5000 & 0.2 & 150 & 3813.8 & 15.74 & 0.067 \\
13 & Multifork I & 5500 & 0.1 & 200 & 3335.29 & 12.66 & 0.065 \\
14 & Multifork II & 5500 & 0.15 & 100 & 1987.52 & 8.96 & 0.063 \\
15 & Standard & 5500 & 0.2 & 150 & 2799.22 & 10.54 & 0.178 \\
16 & Multifork II & 6000 & 0.1 & 200 & 1818.54 & 7.9 & 0.101 \\
17 & Standard & 6000 & 0.15 & 150 & 2411.55 & 10.54 & 0.133 \\
18 & Multifork I & 6000 & 0.2 & 100 & 3971.78 & 16.92 & 0.102 \\
\hline
\end{tabular}


Table 3. Convergence performances for different back propagation wavelet neural network architectures

\begin{tabular}{llllllll}
\hline $\begin{array}{l}\text { Neural network } \\
\text { Architecture }\end{array}$ & $\begin{array}{l}\text { Momentum } \\
\text { Coefficient }(\alpha)\end{array}$ & $\begin{array}{l}\text { Learning rate } \\
(\eta)\end{array}$ & $\begin{array}{l}\text { MSE } \\
\text { Training }\end{array}$ & $\begin{array}{l}\text { MSE } \\
\text { Testing }\end{array}$ & $\begin{array}{l}\text { Number of } \\
\text { Iterations }\end{array}$ & $\begin{array}{l}\text { Maximum predicted } \\
\text { Error }(\%)\end{array}$ & $\begin{array}{l}\text { Minimum predicted } \\
\text { Error }(\%)\end{array}$ \\
\hline $5-5-1$ & 0.9 & 0.8 & 0.0000297 & 0.0000088 & 98 & 9.38 & 0.000774 \\
$5-5-1$ & 0.8 & 0.6 & 0.0000291 & 0.0000068 & 143 & 9.14 & 0.0044 \\
$5-5-1$ & 0.6 & 0.4 & 0.0000297 & 0.0000069 & 280 & 9.43 & 0.00582 \\
$5-5-1$ & 0.5 & 0.3 & 0.0000298 & 0.0000131 & 316 & 9.42 & 0.00583 \\
$5-10-1$ & 0.9 & 0.8 & 0.0000291 & 0.0000118 & 55 & 9.12 & 0.000527 \\
$5-10-1$ & 0.8 & 0.6 & 0.0000294 & 0.0000169 & 82 & 9.27 & 0.00146 \\
$5-10-1$ & 0.6 & 0.4 & 0.0000294 & 0.0000089 & 140 & 9.26 & 0.00226 \\
$5-10-1$ & 0.5 & 0.3 & 0.0000295 & 0.0000023 & 227 & 9.58 & 0.00667 \\
$5-15-1$ & 0.9 & 0.8 & 0.0000274 & 0.0000051 & 38 & 9.18 & 0.000733 \\
$5-15-1$ & 0.8 & 0.6 & 0.0000278 & 0.0000227 & 68 & 9.39 & 0.000636 \\
$5-15-1$ & 0.6 & 0.4 & 0.0000290 & 0.0000028 & 132 & 9.33 & 0.00902 \\
$5-15-1$ & 0.5 & 0.3 & 0.0000294 & 0.0000067 & 168 & 9.15 & \\
\hline
\end{tabular}

Table 4. Convergence performance for different back propagation neural network architectures

\begin{tabular}{llllllll}
\hline $\begin{array}{l}\text { Neural network } \\
\text { Architecture }\end{array}$ & $\begin{array}{l}\text { Momentum } \\
\text { Coefficient }(\alpha)\end{array}$ & $\begin{array}{l}\text { Learning rate } \\
(\eta)\end{array}$ & $\begin{array}{l}\text { MSE } \\
\text { Training }\end{array}$ & $\begin{array}{l}\text { MSE } \\
\text { Testing }\end{array}$ & $\begin{array}{l}\text { Number of } \\
\text { Iterations }\end{array}$ & $\begin{array}{l}\text { Maximum predicted } \\
\text { Error }(\%)\end{array}$ & $\begin{array}{l}\text { Minimum predicted } \\
\text { Error }(\%)\end{array}$ \\
\hline $5-5-1$ & 0.9 & 0.8 & 0.0000483 & 0.0000064 & 52 & 12.31 & 0.00233 \\
$5-5-1$ & 0.8 & 0.6 & 0.0000481 & 0.0000523 & 75 & 11.42 & 0.0148 \\
$5-5-1$ & 0.6 & 0.4 & 0.0000490 & 0.0000029 & 126 & 11.39 & 0.0112 \\
$5-5-1$ & 0.5 & 0.3 & 0.0000487 & 0.0000396 & 183 & 10.19 & 0.0172 \\
$5-8-1$ & 0.9 & 0.8 & 0.0000482 & 0.0000095 & 64 & 10.16 & 0.00188 \\
$5-8-1$ & 0.8 & 0.6 & 0.0000478 & 0.0000285 & 74 & 11.29 & 0.009360 \\
$5-8-1$ & 0.6 & 0.4 & 0.0000492 & 0.0000289 & 128 & 10.55 & 0.00202 \\
$5-8-1$ & 0.5 & 0.3 & 0.0000483 & 0.0000053 & 243 & 14.47 & 0.00147 \\
$5-12-1$ & 0.9 & 0.8 & 0.0000422 & 0.0000046 & 201 & 14.88 & 0.000247 \\
$5-12-1$ & 0.8 & 0.6 & 0.0000423 & 0.0000039 & 358 & 15.08 & 0.000430 \\
$5-12-1$ & 0.6 & 0.4 & 0.0000496 & 0.0000045 & 375 & 7.26 & 0.000508 \\
$5-12-1$ & 0.5 & 0.3 & 0.0000456 & 0.0000049 & 563 & 8.48 & 0.000443 \\
\hline
\end{tabular}

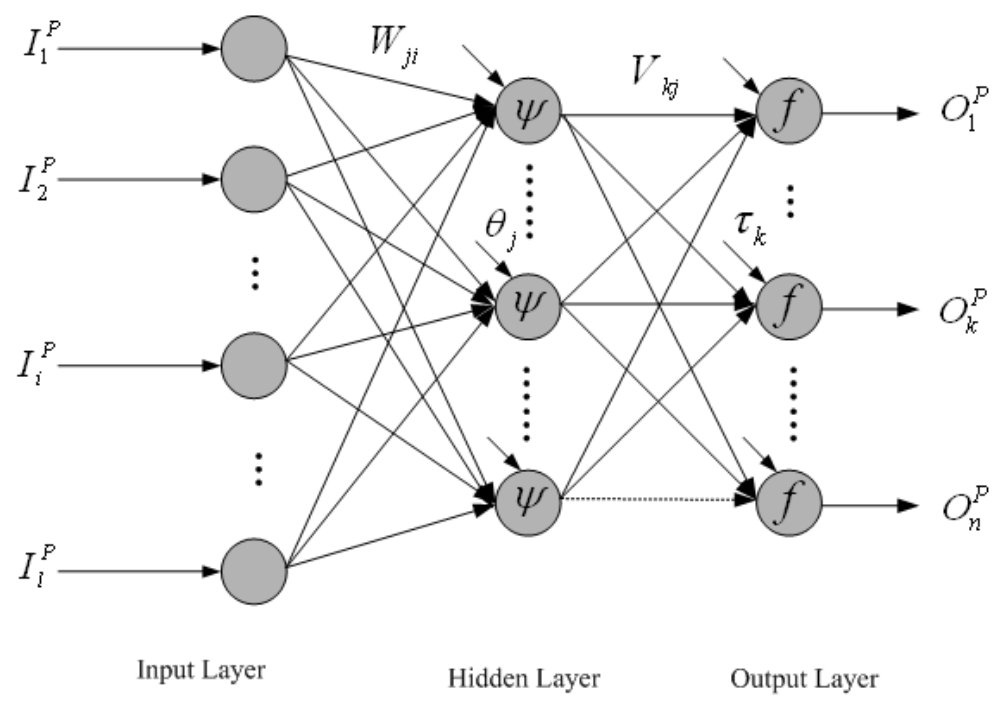

Figure 1. Schematic diagram of Neural network with nine input nodes and one output node 


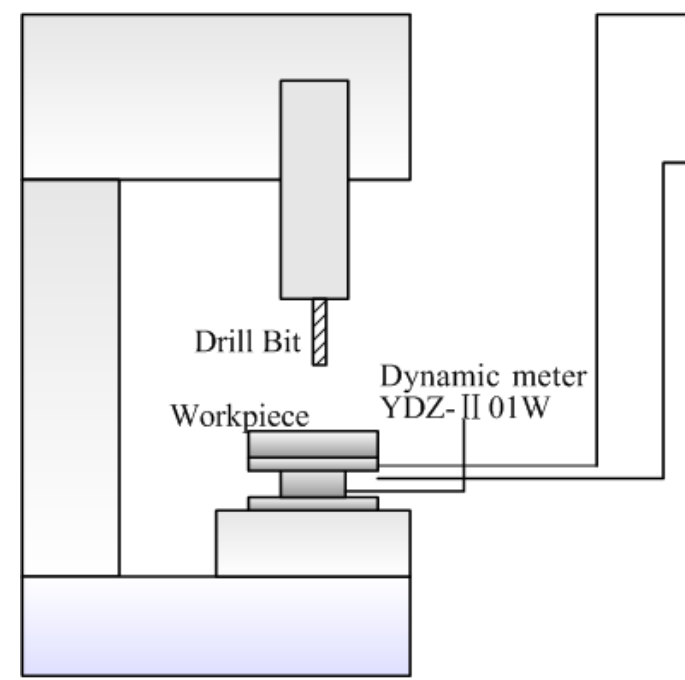

Drilling $\mathrm{CNC}$

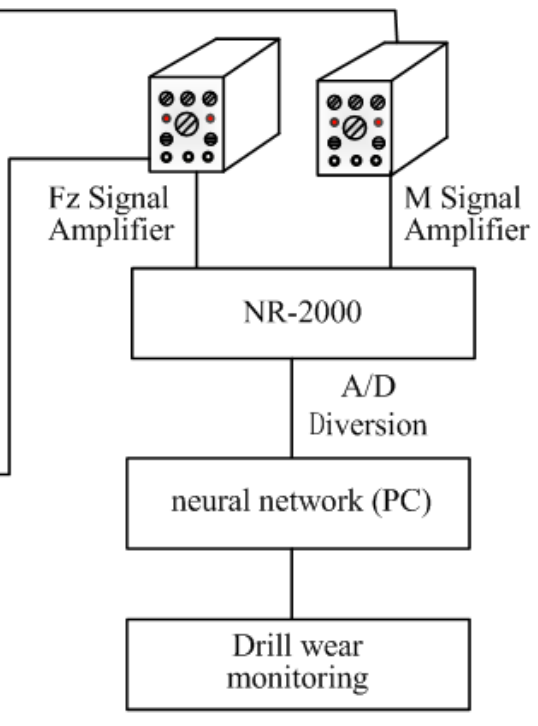

Figure 2. Schematic diagram of the experimental set-up

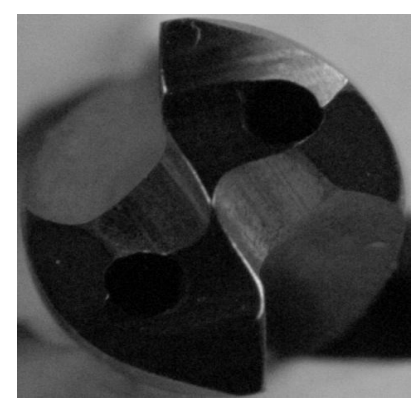

(a)

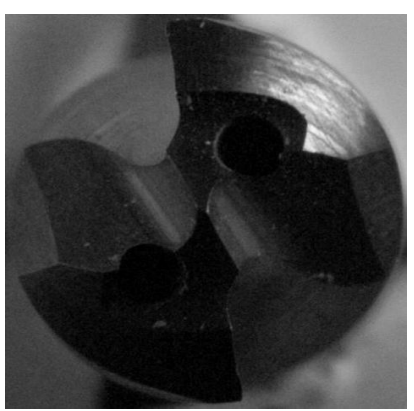

(b)

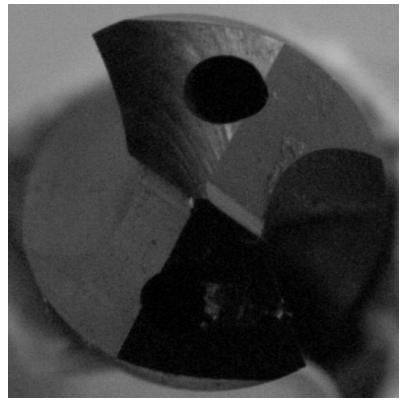

(c)

Figure 3. Flank of standard and Multifacet drill

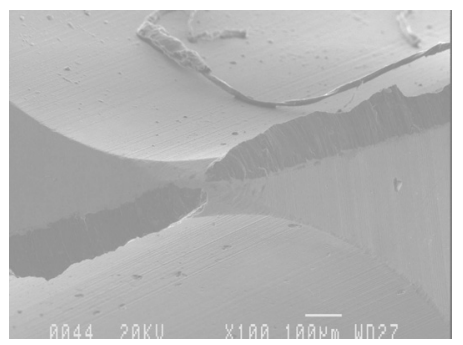

(a).

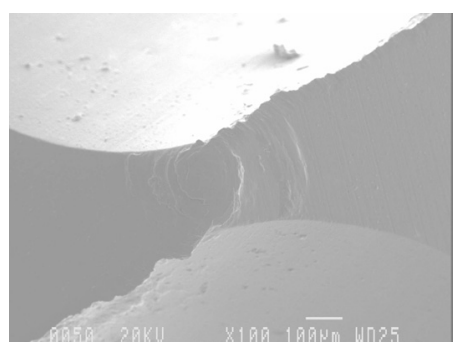

(b)

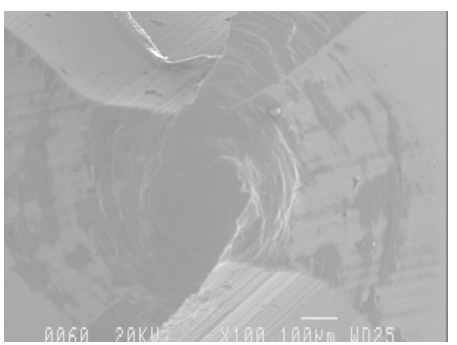

(c)

Figure 4. (a) Drill wear of Multifacet I at spindle speed $4500 \mathrm{rpm}$ and feed rate $0.1 \mathrm{~mm} / \mathrm{rev}$. (b) Drill wear of Multifacet II at spindle speed $4500 \mathrm{rpm}$ and feed rate $0.15 \mathrm{~mm} / \mathrm{rev}$.

(c) Drill wear of standard at spindle speed $4500 \mathrm{rpm}$ and feed rate $0.2 \mathrm{~mm} / \mathrm{rev}$. 

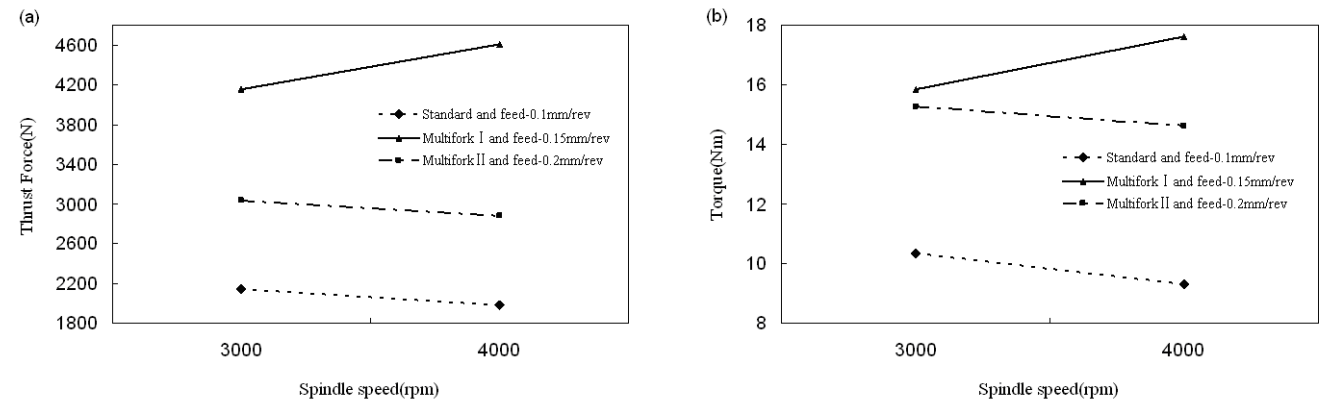

Figure 5. (a) Variation of average thrust force with spindle speed (3000 and 4000rpm) for different feed rates and drills flank. (b) Variation of average torque with spindle speed (3000 and 4000rpm) for different feed rates and drills flank.
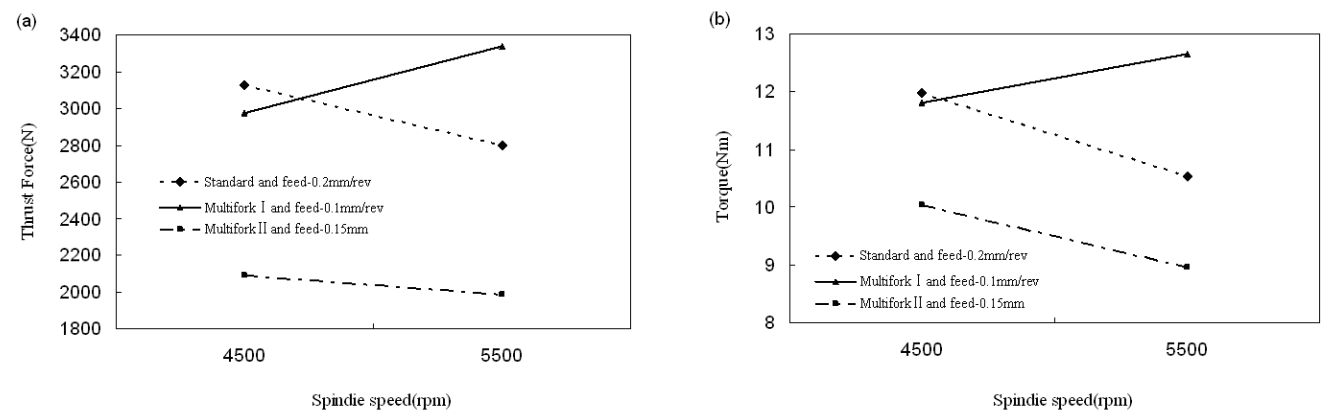

Figure 6. (a) Variation of average thrust force with spindle speed (4500 and 5500rpm) for different feed rates and drills flank. (b) Variation of average torque with spindle speed (4500 and 5500rpm) for different feed rates and drills flank.
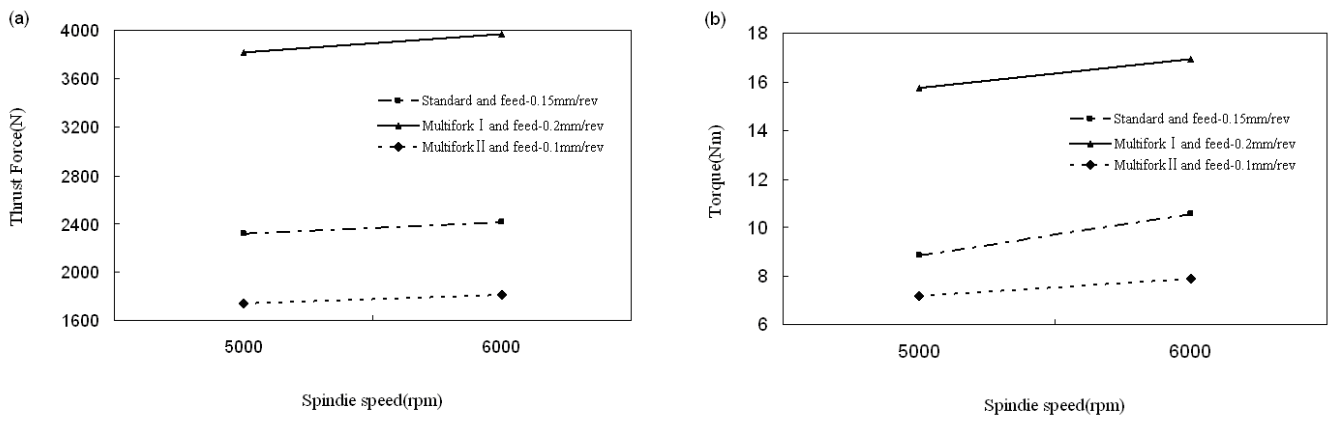

Figure 7. (a) Variation of average thrust force with spindle speed (5000 and 6000rpm) for different feed rates and drills flank. (b) Variation of average torque with spindle speed (5000 and 6000rpm) for different feed rates and drills flank.
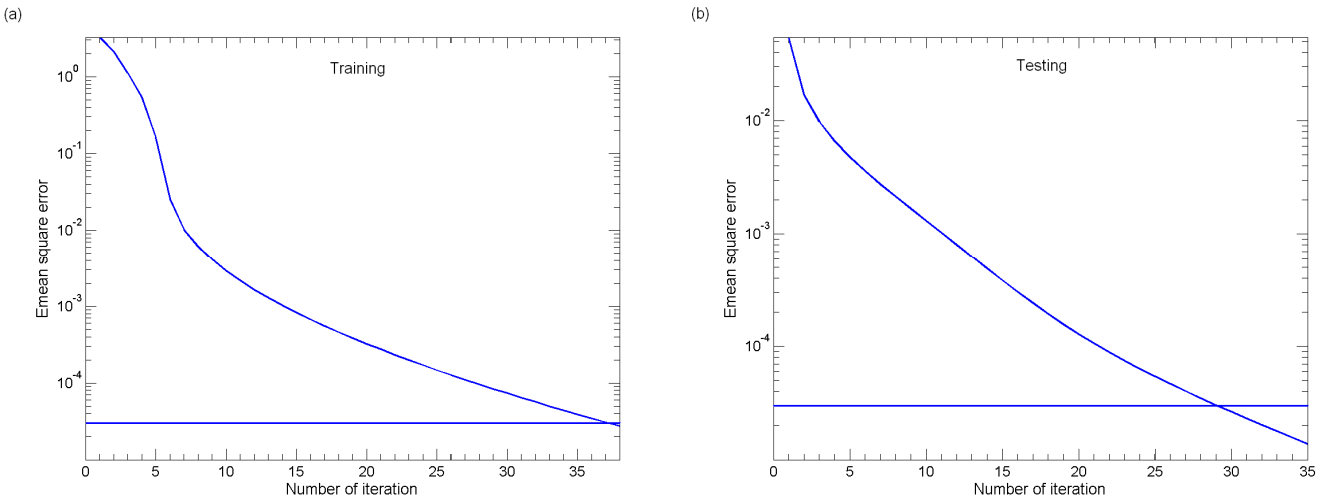

Figure 8 (a). Variation of MSE with number of iteration for the for 5-15-1 neural network with $\alpha=0.9$ and $\eta=0.8$ (BPWNN). (b) Variation of MSE with number of iteration for the for 5-15-1 neural network with $\alpha=0.9$ and $\eta=0.8$ (BPWNN) 
(a)

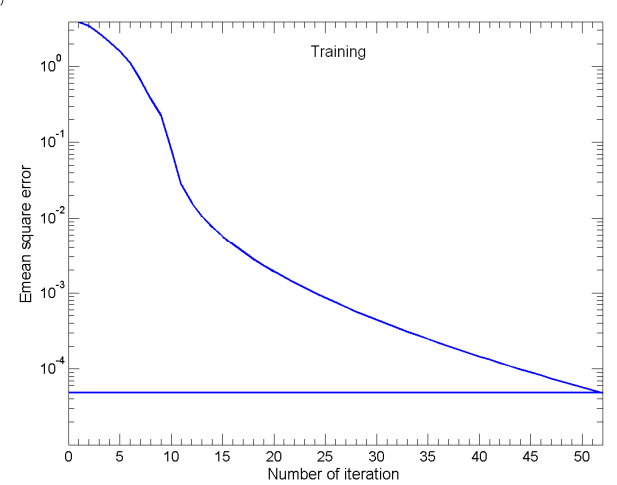

(b)

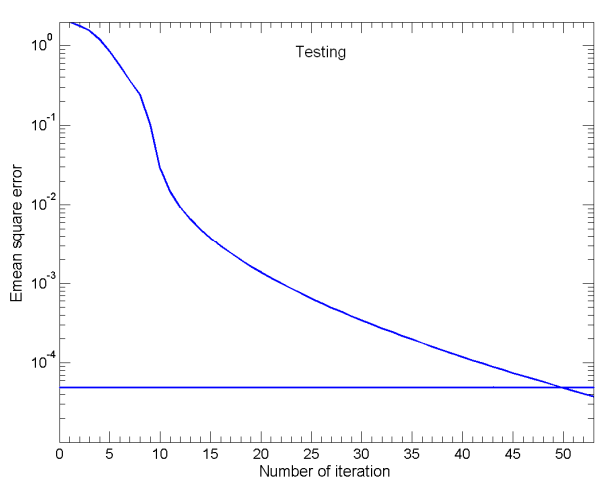

Figure 9(a). Variation of MSE with number of iteration for the for 5-5-1 neural network with $\alpha=0.9$ and $\eta=0.8$ (BPNN). (b) Variation of MSE with number of iteration for the for 5-5-1 neural network with $\alpha=0.9$ and $\eta=0.8(\mathrm{BPNN})$

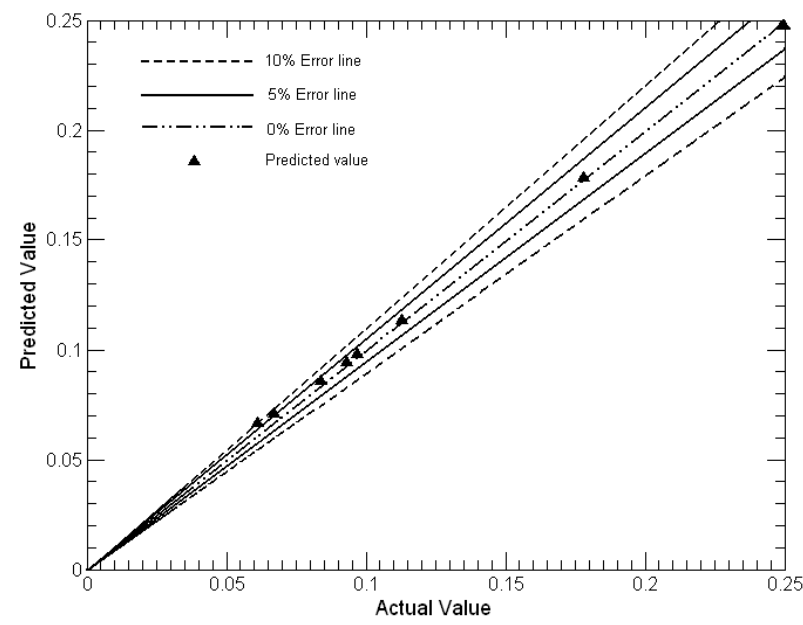

Figure 10. Comparison of predicted values with actual values for 5-15-1 Back propagation wavelet neural network with $\alpha=0.9$ and $\eta=0.8$.

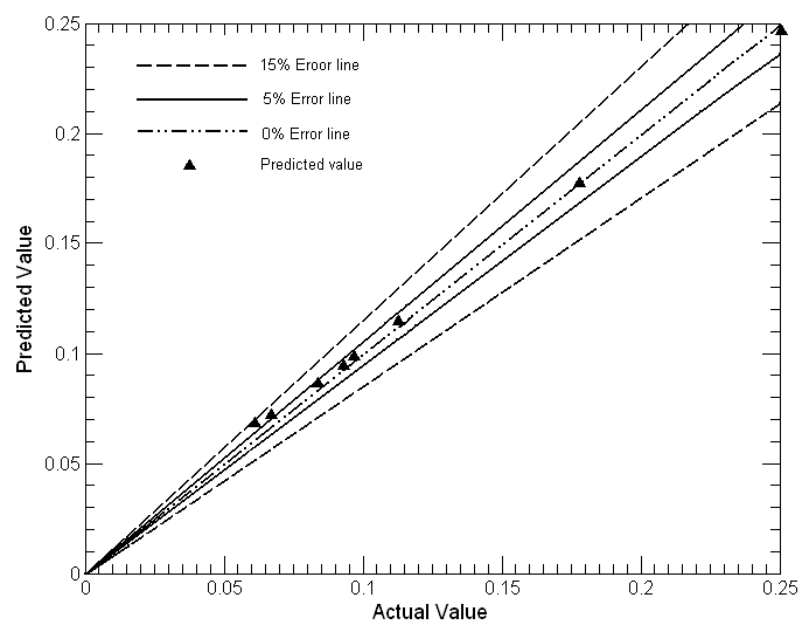

Figure 11. Comparison of predicted values with actuals value for 5-5-1 Back propagation neural network with $\alpha=0.9$ and $\eta=0.8$. 


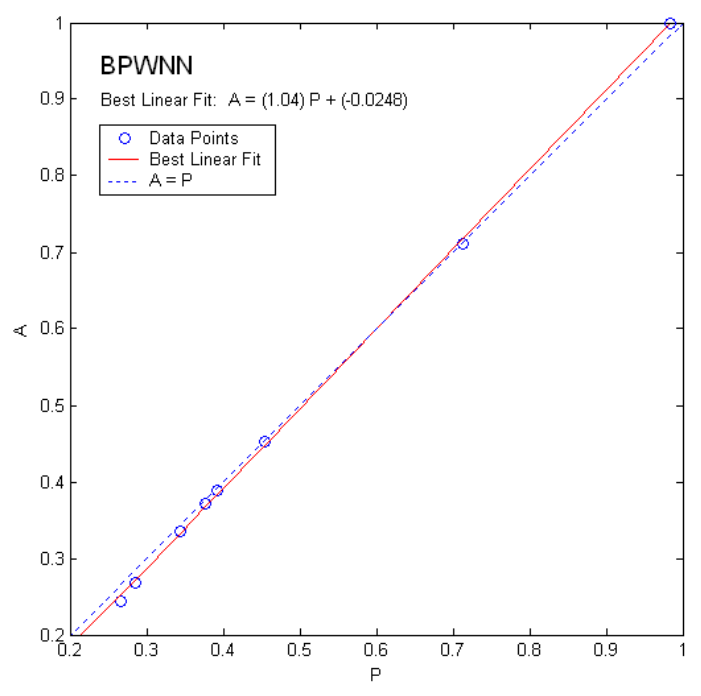

(a)

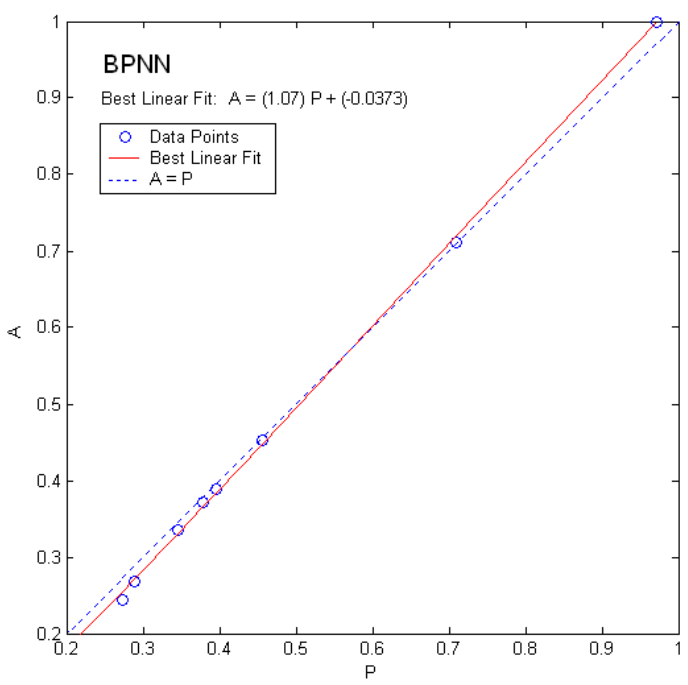

(b)

Figure 12. Comparison between the predictions of drills wear by two models, i.e. (a) BPWNN and (b) BPNN 